\title{
Metachronicity in colon cancer: time to refine our search for follow-up optimization
}

Colorectal cancer is the most common malignancy in Spain. Most cases are sporadic (70-75\%), and hereditary cases represent $3-5 \%$. That the primary prognostic factor for colorectal cancer is disease stage at diagnosis it is a well-known fact being survival at 5 years around $50-55 \%$, raising up to $75-90 \%$ in early stages (1).

The causes of synchronic or metachronic neoplastic lesion development in colorectal cancer is poorly known. Few studies to date have identified the potential risk factors that might determine the development of these multicentric lesions. These studies have yielded conflicting results. In a previous study, Borda et al. retrospectively analyzed 382 diagnosed, surgically excised CRCs, and performed a univariate and multivariate analysis which showed that parameters such as male gender, age older than 59 years, a personal history of colonic polyps, and the cancer's obstructive nature were significantly associated with multicentricity (2). Ballesté et al., members of the renown Spanish gastrointestinal oncology group within Asociación Española de Gastroenterología, have reported a prospective, multicenter, general population-based study of 353 patients with CRC who underwent follow-up colonoscopies at 2 years. In this study, which included an analysis of demographic, clinical, pathological, molecular (microsatellite instability and immunohistochemistry for MSH2 and MLH1), and familial characteristics predictive factors were identified in association with an increased risk for metachronic neoplasia. The univariate analysis demonstrated that metachronic tumor development was associated with a personal history of CRC (OR 5.58, 95\% CI: 1.01-31.01) and the presence of previous or synchronic adenomas (OR 1.77, 95\% CI: 1.21-3.17). However, gender and differentiation degree displayed an association with metachronicity that was not statistically significant. Furthermore, neither microsatellite instability in tumor DNA nor family history were correlated with the presence of metachronic neoplasia (3).

In this issue of Revista Española de Enfermedades Digestivas Borda et al. address this subject again with the authority of a twocenter, highly prestigious group particularly interested in this challenging topic (4). This new manuscript reports on a comparative study of the incidence of metachronic lesions with the characteristic feauture of including a wider number of variables versus previous research. Specifically, it includes 40 patients -and initial tumor-related variables, including molecular markers. The goal of this study was to identify potential risk factors for the development of metachronic lesions in CRC, thus allowing an individual prognostic index to be estimated from the multivariate analysis. In all, 382 patients with resected CRC were studied. Among the 40 parameters studies, 5 showed significant differences in the frequency of metachronic lesion development, and may be considered independent risk factors: distal tumor location, alcohol use, presence of single or multiple synchronic adenomas, advanced synchronic adenoma, and tissue MUC-5 expression, the latter as protective 
parameter. From the score assigned to each variable 3 risk groups were established for the development of metachronic neoplasia, with a frequency of metachronic lesions of $15.8 \%$ in the low-risk group and of $75.3 \%$ in the high-risk group, and non-conclusive results in the medium-risk group. The dichotomization of cases into high and low risk for metachronicity has shown a prognostic sensitivity of $75 \%$ and a specificity of $84 \%$, with a NPV of $92 \%$ and a diagnostic accuracy of $81 \%$. In summary, estimating an individual prognostic index allows an adequate stratification of the risk for metachronic cancer. In this way, a clustering of patients according to their foreseeable risk level will facilitate a better post-operative follow-up. This contribution is relevant, particularly today when colonoscopy adequacy must be maximized in the follow-up of both endoscopically resected adenomas and surgically resected cancer. Endoscopic efforts, with their increased activities and costs, which result from effective screening endoscopies in the general population, should be offset by a decrease in inappropriate colonoscopies. And inadequacy is known to be particularly relevant in this type of follow-up examinations (5). Based on all the above mentioned, the efforts to provide evidence for follow-up guidelines are very important. An added value of the contribution by Borda et al. is the originality of their individual prognostic index for metachronic neoplasia in the setting of CRC, which was not reported to date. That is, it introduces the very interesting notion of high-efficiency follow-up for the future using patient-tailored criteria.

\section{Akiko Ono and Fernando Carballo \\ Unidad de Gestión Clínica de Digestivo. Hospital Universitario Virgen de la Arrixaca. El Palmar, Murcia. Spain}

\section{REFERENCES}

1. Ciccolallo L, Capocaccia R, Coleman MP, Berrino F, Coebergh JW, Damhuis RA, et al. Survival differences between European and US patients with colorectal cancer: role of stage at diagnosis and surgery. Gut 2005;54:268-73.

2. Borda A, Martínez-Peñuela JM, Muñoz-Navas M, Prieto C, Betés M, Borda F. Synchronous neoplastic lesions in colorectal cancer. An analysis of possible risk factors favouring presentation. Rev Esp Enferm Dig 2008;100:139-45.

3. Ballesté B, Bessa X, Piñol V, Castellví-Bel S, Castells A, Alenda C, et al., for the Gastrointestinal Oncology Group of the Spanish Gastroenterological Association. Detection of metachronous neoplasms in colorectal cancer patients: identification of risk factors. Dis Colon Rectum 2007;50:1-10.

4. Borda A, Martínez-Peñuela JM, Borda F, Muñoz-Navas, Jiménez FJ, Carretero C. Drawing up an individual risk index for development of metachronous neoplastic lesions in resected colorectal cancer. Rev Esp Enferm Dig 2012;104(6):291-7

5. Carrión S, Marín I, Lorenzo-Zúñiga V, Moreno de Vega V, Boix J. Adecuación de la indicación de la colonoscopia según los nuevos criterios de EPAGE II. Gastroenterol Hepatol 2010;33:484-9. 